\title{
A meta-analysis on anterior cruciate ligament reconstruction: Is modified transtibial technique inferior to independent drilling techniques?
}

\author{
QUNHU ZHANG ${ }^{1 *}$, YU KOU ${ }^{2 *}$ and ZHEN YUAN ${ }^{3}$ \\ ${ }^{1}$ Department of Orthopedics, Shuyang Hospital of Traditional Chinese Medicine Affiliated to Nanjing University \\ of Chinese Medicine, Suqian Jiangsu 223600; ${ }^{2}$ Medical College of Soochow University, Suzhou Jiangsu 215123; \\ ${ }^{3}$ Center of Sports Medicine and Rehabilitation, The Affiliated Suzhou Hospital \\ of Nanjing Medical University, Suzhou, Jiangsu 215001, P.R. China
}

Received January 18, 2018; Accepted June 26, 2018

DOI: $10.3892 /$ etm.2018.6395

\begin{abstract}
This report respectively compared the standard transtibial (sTT) technique to the independent drilling (ID) techniques applied to anterior cruciate ligament (ACL) reconstruction. It also made a comparison between the clinical results of the modified transtibial (mTT) technique and of the ID techniques. Prospective studies on transtibial (TT) and ID techniques for ACL reconstruction were retrieved from several databases and a subgroup analysis was performed to compare the sTT technique with the ID techniques and the mTT with the ID techniques. Furthermore, comparison of the Lachman test, pivot-shift test, International Knee Documentation Committee (IKDC) subjective and objective evaluations, Lysholm score and Tegner activity scale were conducted. This report included 12 clinical studies that involved 681 patients having received ACL reconstruction. The study results indicated that in comparison between the sTT and ID techniques, the ID techniques outperformed the sTT technique in the IKDC subjective score $(\mathrm{P}=0.01)$ and laxity $(\mathrm{P}=0.0004)$. However, there was no significant difference in the IKDC objective score $(\mathrm{P}=0.34)$, pivot-shift test $(\mathrm{P}=0.24)$, Lachman test $(\mathrm{P}=0.21)$, Lysholm score $(\mathrm{P}=0.14)$ and Tegner activity scale $(\mathrm{P}=0.66)$. The comparison between the new mTT technique and the ID techniques suggested no significant difference in the IKDC objective and subjective scores $(\mathrm{P}=0.86)$, laxity $(\mathrm{P}=0.38)$, pivot-shift test $(\mathrm{P}=0.66)$, Lachman test $(\mathrm{P}=0.10)$, Lysholm score $(\mathrm{P}=0.10)$ and Tegner activity
\end{abstract}

Correspondence to: Dr Zhen Yuan, Center of Sports Medicine and Rehabilitation, The Affiliated Suzhou Hospital of Nanjing Medical University, 242 Guangji Road, Suzhou, Jiangsu 215001, P.R. China E-mail: yz1984312@163.com

*Contributed equally

Key words: anterior cruciate ligament, modified transtibial technique, anatomic reconstruction, meta-analysis scale $(\mathrm{P}=0.55)$. Compared to the sTT technique, the mTT and ID techniques are more suitable for ACL reconstruction because they can present better subjective feelings. Moreover, considering that the TT technique is familiar to surgeons and the mTT technique can bring favorable subjective feelings and objective clinical outcomes, the mTT technique shows greater utilization potential.

\section{Introduction}

Conservative treatment for anterior cruciate (ACL) ligament has modest efficacy. A study (1) suggested that $95 \%$ of the patients who had adopted long-term conservative treatment had to receive meniscectomy after 20 years while $52 \%$ of these patients, at the average age of 53 only, had to receive total knee arthroplasty (TKA) after 30 years; moreover, because conservative treatment may further affect the stability of lateral knee joint, in most cases, surgery is recommended. In the USA, there are $>100,000$ patients receiving ACL reconstruction every year $(2,3)$. Presently, arthroscopic ACL reconstruction techniques mainly include: transtibial (TT), anteromedial portal (AMP) or transportal (TP) and outside-in (OI) techniques. The TT technique means drilling a tibial tunnel to create a femoral tunnel in ACL reconstruction while the AMP and the $\mathrm{OI}$ techniques require an additional incision on the anteromedial or OI aspect of the femur to create a solitary femoral tunnel. Similar to other literature (4), the AMP and the OI techniques are collectively referred to as the independent drilling (ID) techniques in this report. Plenty of studies showed that femoral tunnels could not be precisely placed in the natural ACL anatomic insertion by using the standard transtibial (sTT) technique $(5,6)$, which, as a result, caused abnormal rotation of knee joints $(7,8)$ and failed to reduce the occurrence of knee osteoarthritis (KOA). At present, the sTT technique has been basically abandoned by surgeons. In contrast, the ID techniques are efficient in the placement of femoral tunnel in the natural ACL insertion for anatomic reconstruction. Since the ID techniques have overcome the deficiencies of the sTT technique, it has become a trend to employ the ID 
techniques in ACL reconstruction (9-11). However, in recent years, a new technique has been introduced to the field, the modified transtibial (mTT) technique. The mTT technique is not only as efficacious as the ID techniques in respect of anatomic reconstruction $(4,12,13)$, but requires fewer incisions compared to the latter. Moreover, most surgeons in the field of sports medicine have grasped the TT technique and thus they are likely to acknowledge the mTT technique. Is the mTT technique or the ID techniques better for ACL reconstruction? This report gives a meta-analysis of the latest studies on the sTT, ID and mTT techniques, which evaluated these techniques by comparing their clinical data and provided a theoretical basis for surgeons to select the optimal surgical methods.

\section{Data collection methods}

Search strategy and study selection. We conducted online searches of PubMed (1982 to April 2017), Cochrane (4th issue, 2017) and Embase (1982 to June 2017), the following words were selected to perform the search task in the PubMed, Embase and Cochrane databases by means of medical subject headings and text-word searching: 'anterior cruciate ligament' and ('transtibial' or 'modified transtibial' or 'anteromedial portal' or 'outside-in' or 'independent drilling'). Titles and abstracts of the retrieved articles were assessed to exclude the ineligible ones from the meta-analysis. Further inclusion and exclusion was carried out by means of full-text assessment for eligibility.

The inclusion criteria are as follows: i) clinical studies on comparison between the TT (or mTT) and the ID techniques for ACL reconstruction; ii) single-bundle ACL reconstruction; iii) patients suffering from ACL fracture without any other ligament injuries; iv) complete reports on postoperative kinematic and clinical evaluation results; and v) prospective clinical studies (level of evidence: level I and II).

The exclusion criteria are as follows: i) studies on comparison between the TT technique and other drilling methods, or between the sTT and the mTT techniques or the TP and the OI techniques; ii) studies on double-bundle ACL reconstruction; iii) ACL accompanied by posterior cruciate ligament (PCL), medial collateral ligament (MCL) or lateral collateral ligament (LCL) injuries; iv) absence of reports on postoperative clinical outcomes or failure in acquiring necessary data; and v) retrospective clinical studies or those with lower levels of evidence (level III or IV or below).

These criteria were also applied to the references of each article included in this study for screening to prevent careless omission.

Data extraction and analysis. The data extracted from the included articles were: first author, year of publication, research type, level of evidence, average age of patients, sample size, follow-up duration, occurrence of meniscus injury, International Knee Documentation Committee (IKDC) subjective/objective score, laxity of knee joint, Lachman test, pivot-shift test, Lysholm score and Tegner activity scale. When having any questions about an article, the authors of this report tried to reach and communicate with the correspondence author(s) of the article.
Since the TT groups of the included articles either referred to the sTT or the mTT technique, in this study, they were divided into the sTT and the mTT subgroups for comparison with the ID techniques.

RevMan 5.3 software was employed to perform data analysis. The standardized mean differences (SMDs) of continuous variables were used as analytical results while the random effects model with $95 \%$ confidence intervals (CIs) was applied to the analysis; on the other hand, the odds ratios (ORs) of dichotomous variables were adopted to present analytical results and similarly, the random effects model with $95 \%$ CI was applied to the analysis. $\mathrm{I}^{2}$ was used to assess heterogeneity. If $\mathrm{I}^{2}<50 \%$, there was low heterogeneity. $\mathrm{P}<0.05$ suggested a significant difference in analytical results. In some studies (14-17), continuous variables were expressed by medians and numerical ranges. The method put forward by Hozo et al (18) in 2005 was employed to estimate means and variances; some used means and P-values to report continuous variables. In this study, the methods provided in the Cochrane Handbook for Systematic Reviews of Interventions were adopted as variance estimators.

\section{Results}

Identification process. With the above-mentioned key words, 689 articles were retrieved from the PubMed database while 721 were retrieved from the Embase database and 79 were from the Cochrane database. After removing duplicates, there remained 882 records in total. The authors of this report selected 50 articles eligible for the meta-analysis after going through the titles and abstracts of all records. Subsequently, a full-text assessment for eligibility of these 50 articles was performed and 12 studies (Fig. 1) involving 681 patients were included in the meta-analysis according to the foregoing inclusion and exclusion criteria. The first authors, years of publication, research types, levels of evidence, sample sizes, follow-up durations, occurrence of meniscus injury and the involved drilling techniques for comparison of these 12 articles are shown in Table I.

Measurements meta-analysis. The IKDC objective score was analyzed as a dichotomous variable $(16,19-22)$ and the OR value was defined as (number of patients rated A and B)/(number of patients rated $C$ and D)'. There were 305 patients in five studies included in the comparison between the sTT and the ID techniques. Through analysis, there were no statistically significant differences in the IKDC objective scores of the sTT and the ID group [OR=0.48, 95\% CI $(0.11,2.14), \mathrm{P}=0.34]$. The data showed low heterogeneity $\left(\mathrm{P}=0.46, \mathrm{I}^{2}=0 \%\right)$. In addition, based on the comparison of the IKDC objective scores between the mTT and the ID techniques, a study (sample size, 38 ) indicated no statistical differences between the two types of techniques in this respect $[\mathrm{OR}=1.00,95 \% \mathrm{CI}(0.06,17.18)$, $\mathrm{P}=1.00$ ] (23) (Fig. 2).

An analysis on the IKDC subjective score as a continuous variable was performed (14,19-21). There were 257 patients in four studies included in the comparison between the sTT and the ID techniques. The study results suggested statistically significant differences between the sTT group and that of the ID group in their IKDC subjective scores [SMD=-0.48, 
Table I. Basic information of included studies.

\begin{tabular}{|c|c|c|c|c|c|c|c|c|}
\hline Author & Year & $\begin{array}{l}\text { Experimental } \\
\text { design }\end{array}$ & $\begin{array}{l}\text { Level of } \\
\text { evidence }\end{array}$ & $\begin{array}{l}\text { Age in years } \\
\text { mean (range) }\end{array}$ & $\begin{array}{l}\text { Sample } \\
\text { size }\end{array}$ & $\begin{array}{l}\text { Meniscus } \\
\text { injury }\end{array}$ & $\begin{array}{c}\text { Follow-up } \\
\text { duration (months) }\end{array}$ & $\begin{array}{l}\text { Techniques for } \\
\text { comparison }\end{array}$ \\
\hline Matassi et al (21) & 2015 & & II & $31(18-48)$ & 40 & - & 12 & TT/OI \\
\hline Lanzetti et al (14) & 2017 & & II & $25.71 \pm 3.02$ & 44 & + & $>24$ & TT/OI \\
\hline Sohn et al (15) & 2014 & & II & $29.3(15-51)$ & 60 & - & 15.7 & mTT/AMP/OI \\
\hline Zhang et al (25) & 2012 & $\mathrm{RCT}$ & $\mathrm{I}$ & $28(17-48)$ & 76 & - & $>12$ & TT/AMP \\
\hline Noh et al (16) & 2013 & & I & $23(18-45)$ & 61 & - & 30.2 & TT/AMP \\
\hline Musahl (17) & 2015 & & II & 29 & 40 & - & 24 & mTT/AMP \\
\hline Youm et al (23) & 2014 & & $\mathrm{I}$ & $28.7 \pm 10.9$ & 40 & + & 24 & mTT/AMP \\
\hline Hussein et al (20) & 2012 & RCT & I & $33.4(16-63)$ & 150 & - & 51.15 & TT/AMP/AMP(DB) \\
\hline Bohn et al (19) & 2015 & $\mathrm{RCT}$ & I & $25.9 \pm 6.3$ & 23 & - & 13 & TT/AMP \\
\hline Koutras et al (26) & 2013 & & II & 23.9 & 51 & - & 6 & TT/AMP \\
\hline Xu et al (24) & 2012 & & II & $32.0 \pm 4.8$ & 65 & - & 16 & TT/AMP \\
\hline Yanasse et al (22) & 2016 & & II & $18-45$ & 31 & - & 13 & TT/OI \\
\hline
\end{tabular}

RCT, randomized controlled trial; TT, transtibial; OI, outside-in; mTT, modified transtibial; AMP, anteromedial portal.

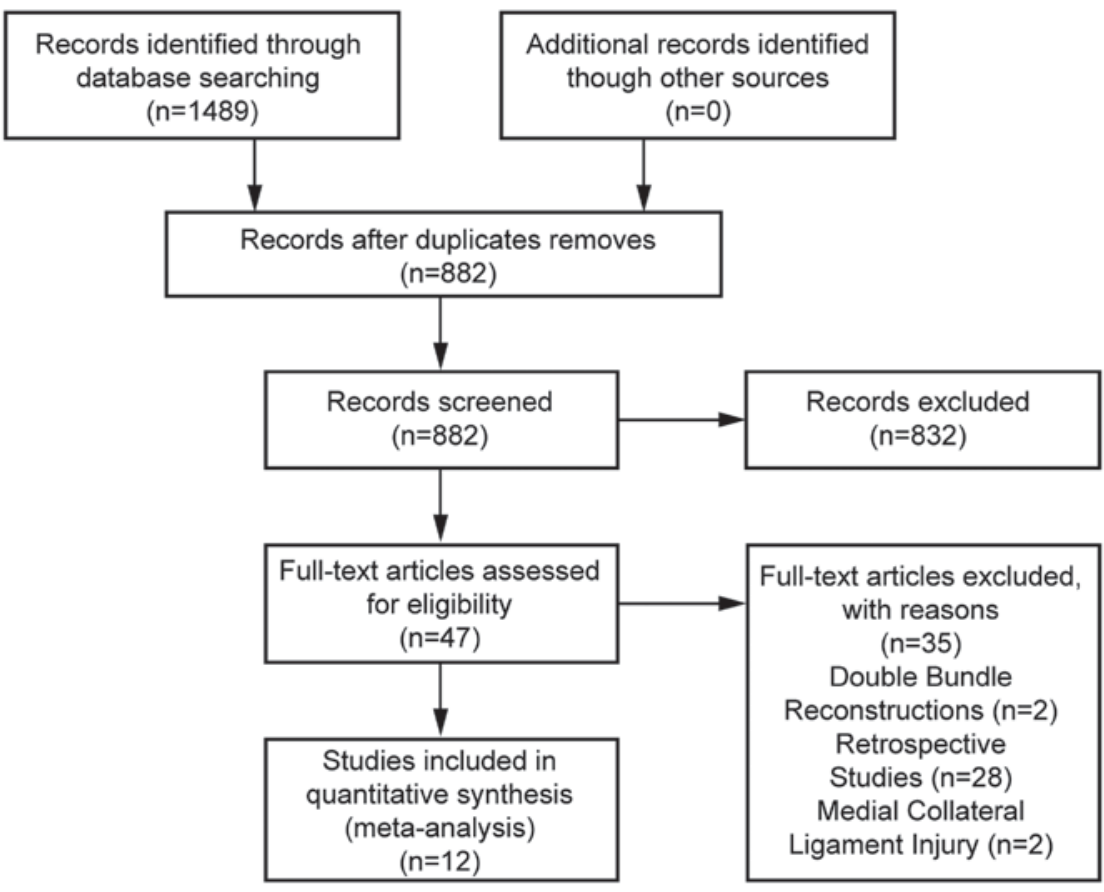

Figure 1. Records screening flow chart.

95\% CI (-0.86,-0.10), $\mathrm{P}=0.01]$, with the ID group having a higher score. The data showed moderate heterogeneity $\left(\mathrm{P}=0.15, \mathrm{I}^{2}=44 \%\right)$. Besides, there were 140 patients in three studies $(15,17,23)$ included in the comparison between the mTT and the ID techniques. According to the study results, there were no statistically significant differences in the IKDC subjective scores of the mTT and the ID group [SMD=-0.22, $95 \%$ CI $(-0.56,0.12), \mathrm{P}=0.20]$. The data showed no heterogeneity $\left(\mathrm{P}=0.86, \mathrm{I}^{2}=0 \%\right)$ (Fig. 3$)$.

The difference in the anterior displacement of the tibia between bilateral knee joints was employed to assess the difference in anterior displacement of the tibia between the operated and the intact knees (14,19-21,24,25). In four studies, the KT-1000 knee arthrometer was adopted while the KT-2000 knee arthrometer was applied to another two studies. The difference in bilateral knee joints was analyzed as a continuous variable. There were 398 patients in six studies included in the comparison between the sTT and the ID techniques. The study results indicated statistically significant differences between the sTT and the ID groups in D-value in anterior displacement of the tibia between the operated and the intact knees [SMD=0.36, 95\% CI $(0.16,0.56), \mathrm{P}=0.0004]$, with the ID group having a smaller D-value. The data showed low heterogeneity $\left(\mathrm{P}=0.82, \mathrm{I}^{2}=0 \%\right)$. Besides, there was a study (sample size, 60) comparing the mean difference in the anterior displacement of the tibia between the operated and 


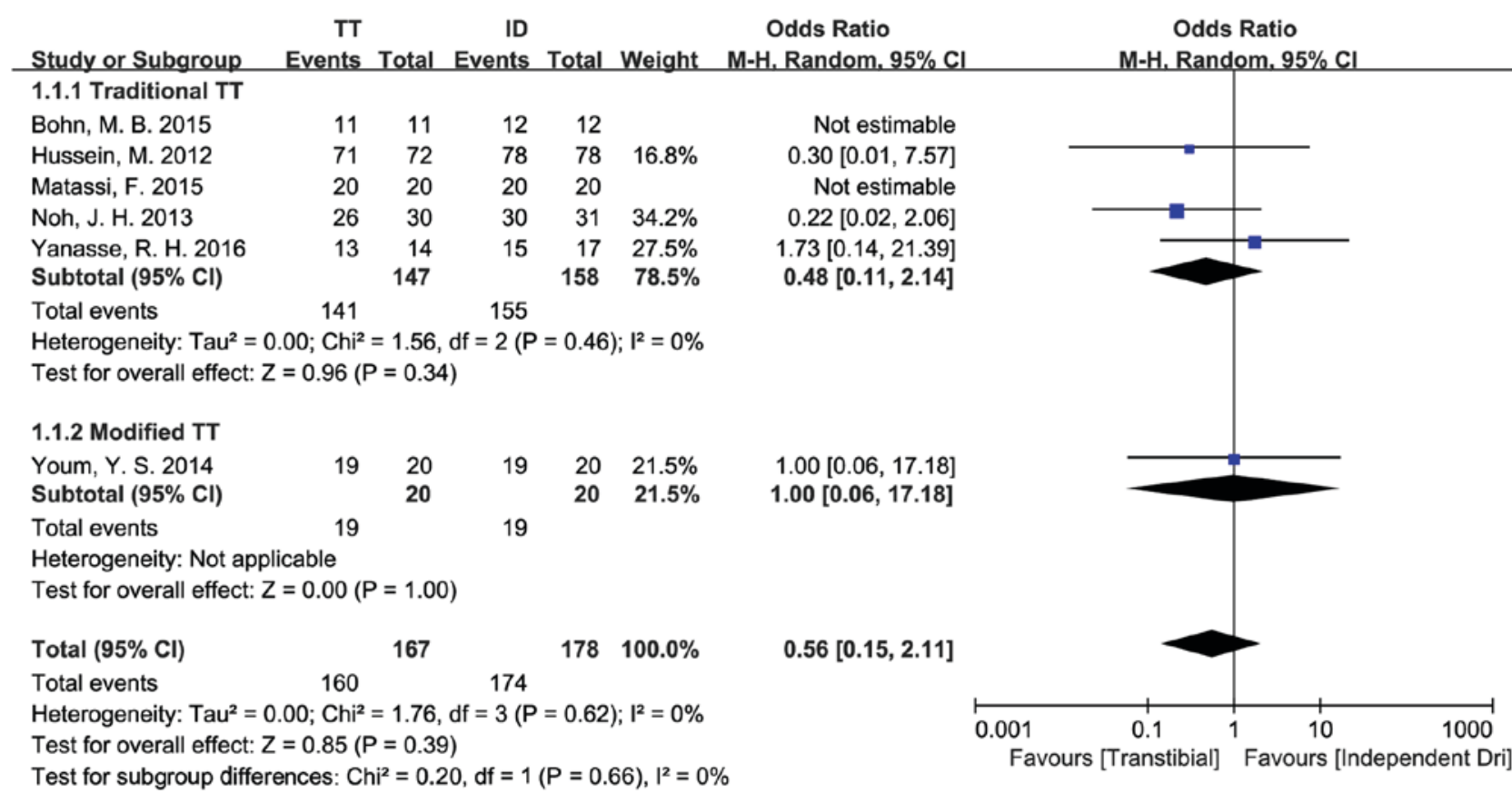

Figure 2. Comparison of IKDC objective scores between the TT and the ID group. IKDC, International Knee Documentation Committee; TT, transtibial; ID, independent drilling.

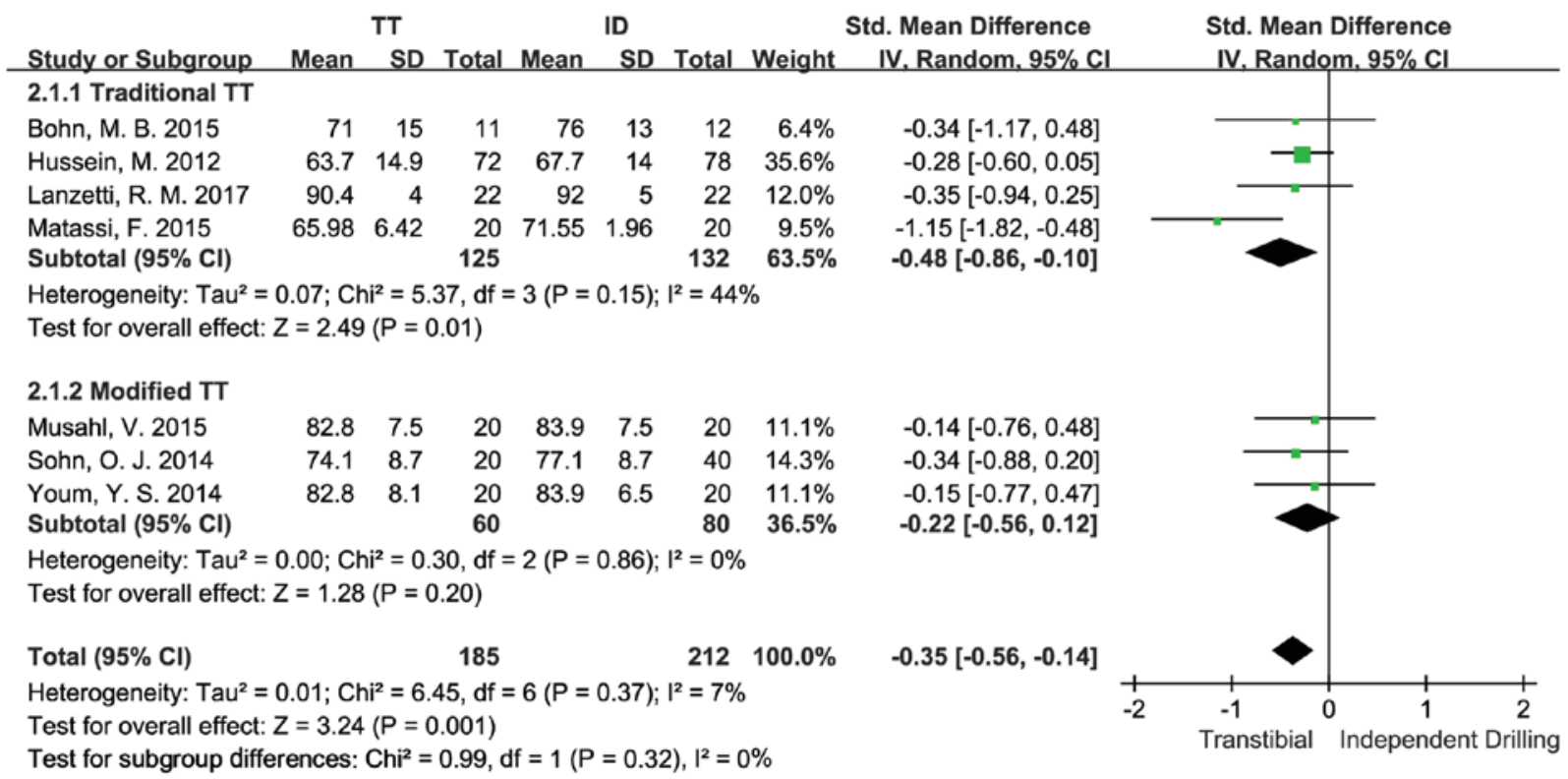

Figure 3. Comparison of IKDC subjective scores between the TT and the ID group. IKDC, International Knee Documentation Committee; TT, transtibial; ID, independent drilling.

the intact knees of the mTT and ID groups (15). From the study results, no statistical differences in this respect between the sTT and the ID techniques were found [OR $=0.24,95 \% \mathrm{CI}$ (-0.30, 0.78), P=0.38] (Fig. 4).

An analysis on the Lachman test results as dichotomous variables was conducted $(14,16,19,22)$ and the OR value was defined as (number of patients with positive test results)/(number of patients with negative test results)'. There were 159 patients in four studies included in the comparison between the sTT and the ID techniques. Through analysis, there were no statistically significant differences in the Lachman test results between the
sTT and the ID group [OR=1.72, 95\% CI $(0.74,3.97), \mathrm{P}=0.21]$. The data showed low heterogeneity $\left(\mathrm{P}=0.34, \mathrm{I}^{2}=7 \%\right)$. There was also a study (sample size, 40) comparing the Lachman test results of the mTT and the ID techniques (23), according to which, no statistical difference between the mTT and the ID techniques was found in Lachman test [OR $=0.47,95 \% \mathrm{CI}$ (0.04, 5.69), $\mathrm{P}=0.56$ ] (Fig. 5).

The pivot-shift test results were analyzed as dichotomous variables $(14,16,19,20,22,24)$ and the OR value was defined as (number of patients with positive test results/number of patients with negative test results). There were 364 patients in six studies 


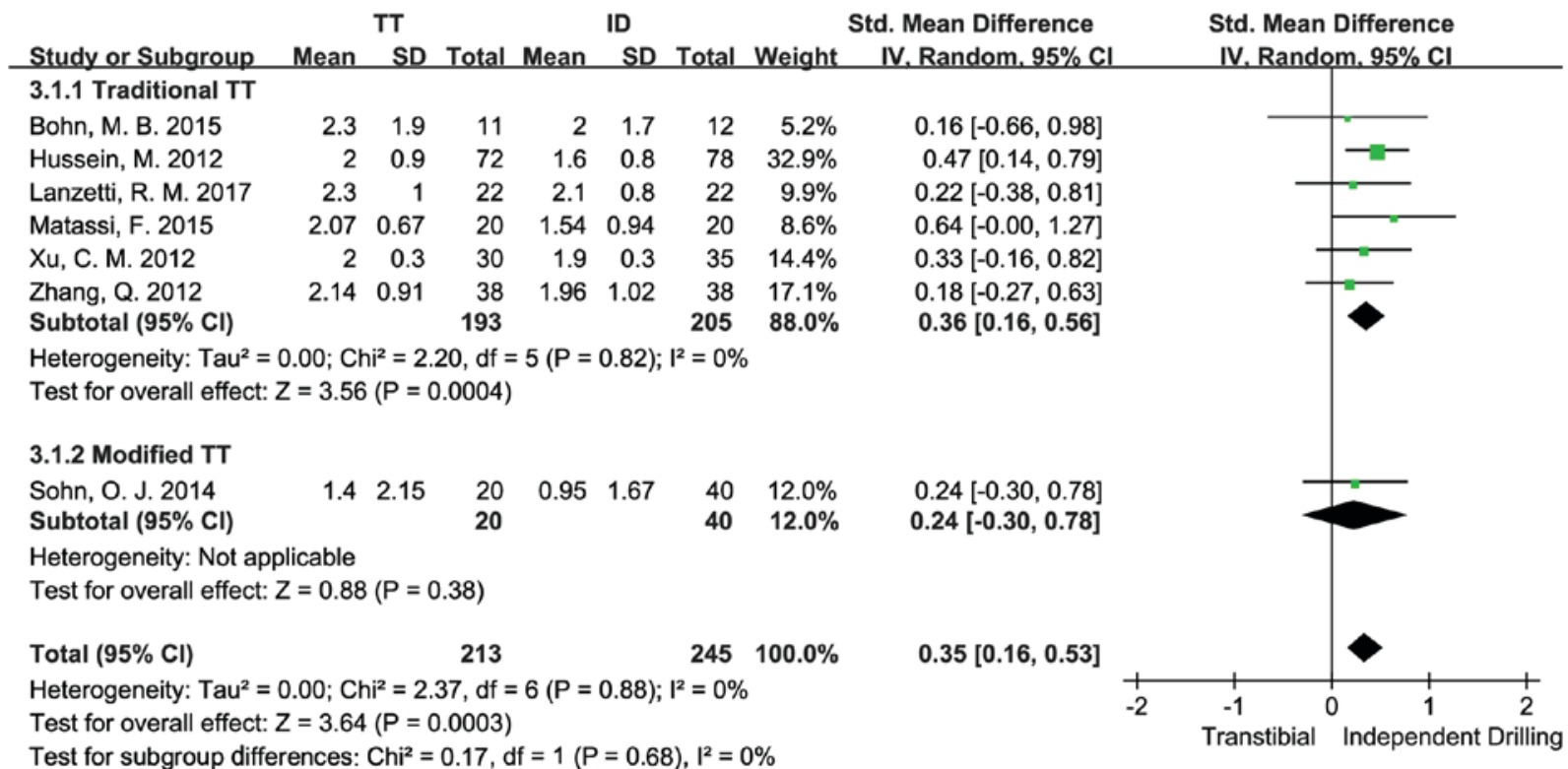

Figure 4. Comparison of differences in laxity of bilateral knee joints between the TT and the ID group. TT, transtibial; ID, independent drilling.

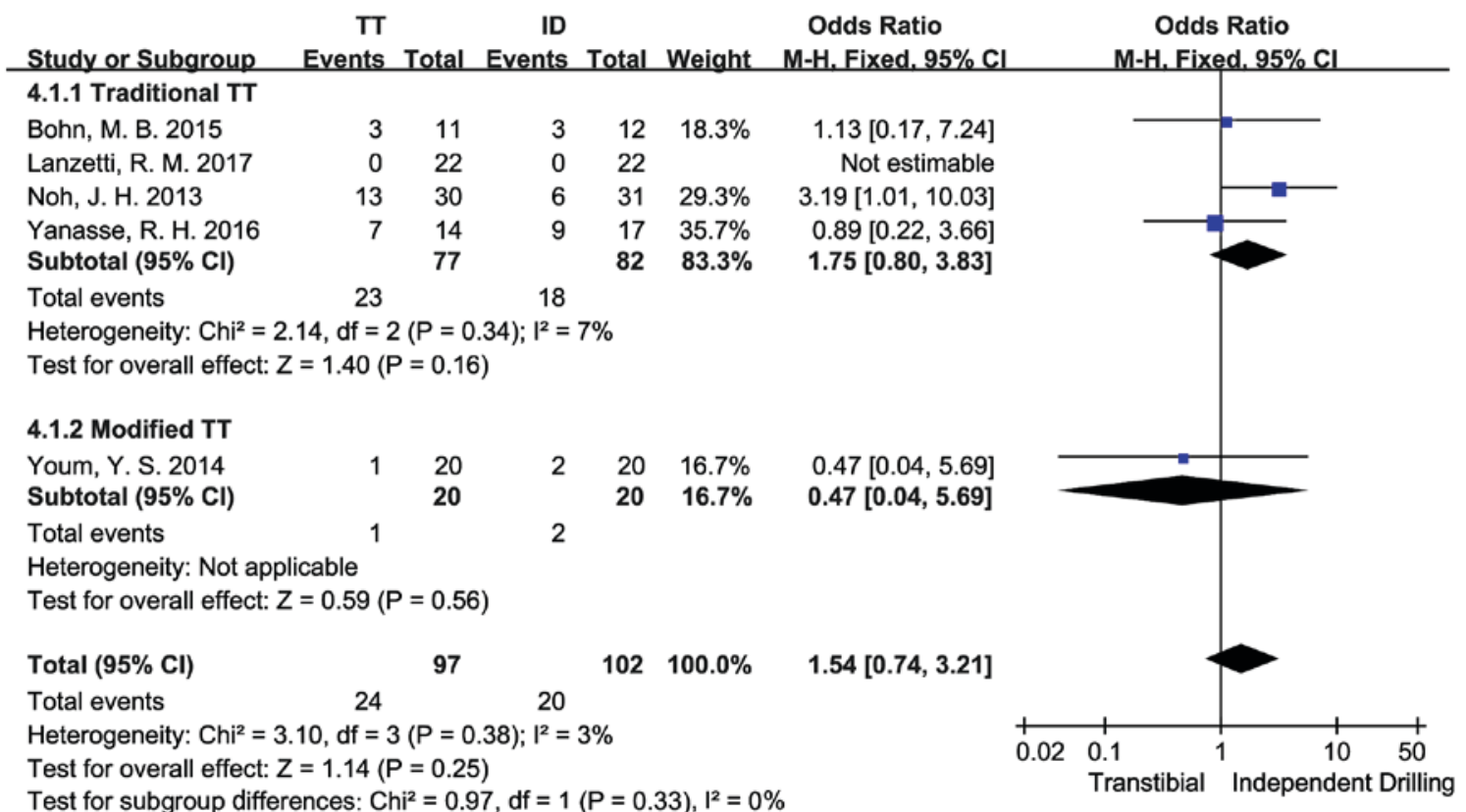

Figure 5. Comparison of Lachman test results between the TT and the ID group. TT, transtibial; ID, independent drilling.

included in the comparison between the sTT and the ID techniques. According to the study results, no statistically significant differences were found in the pivot-shift test results between the sTT group and the ID group [OR $=1.70,95 \%$ CI $(0.70,4.15)$, $\mathrm{P}=0.24]$. The data showed moderate heterogeneity $(\mathrm{P}=0.04$, $\left.\mathrm{I}^{2}=56 \%\right)$. There were 100 patients in two studies $(15,23)$ included in the comparison between the mTT and the ID techniques. The results showed no statistically significant differences in the pivot-shift test results between the mTT and the ID group $[\mathrm{OR}=1.72,95 \% \mathrm{CI}(0.16,18.98), \mathrm{P}=0.66]$. The data showed moderate heterogeneity ( $\left.\mathrm{P}=0.16, \mathrm{I}^{2}=49 \%\right)$ (Fig. 6).

The Lysholm knee score was analyzed as a continuous variable $(14,16,19,20,22,24,25,26)$ in eight articles where a total number of 501 patients were included in the comparison between the sTT and the ID techniques. The results indicated no statistically significant differences in Lysholm knee score between the sTT and the ID group [SMD $=-0.19,95 \% \mathrm{CI}$ $(-0.44,0.06), \mathrm{P}=0.14]$. The data showed a certain degree of heterogeneity within the tolerance interval $\left(\mathrm{P}=0.08, \mathrm{I}^{2}=45 \%\right)$. In addition, there were 140 patients in three studies $(15,17,23)$, included in the comparison between the mTT and the ID techniques. The results suggested that there was no statistically significant difference in Lysholm knee score between the mTT and the ID group [SMD=-0.37, 95\% CI (-0.82, 0.07), $\mathrm{P}=0.10]$ with the data showing moderate heterogeneity $(\mathrm{P}=0.19$, $\mathrm{I}^{2}=40 \%$ ) (Fig. 7).

The Tegner activity scale was analyzed as a continuous variable $(14,19,22)$, in three articles where 98 patients were 


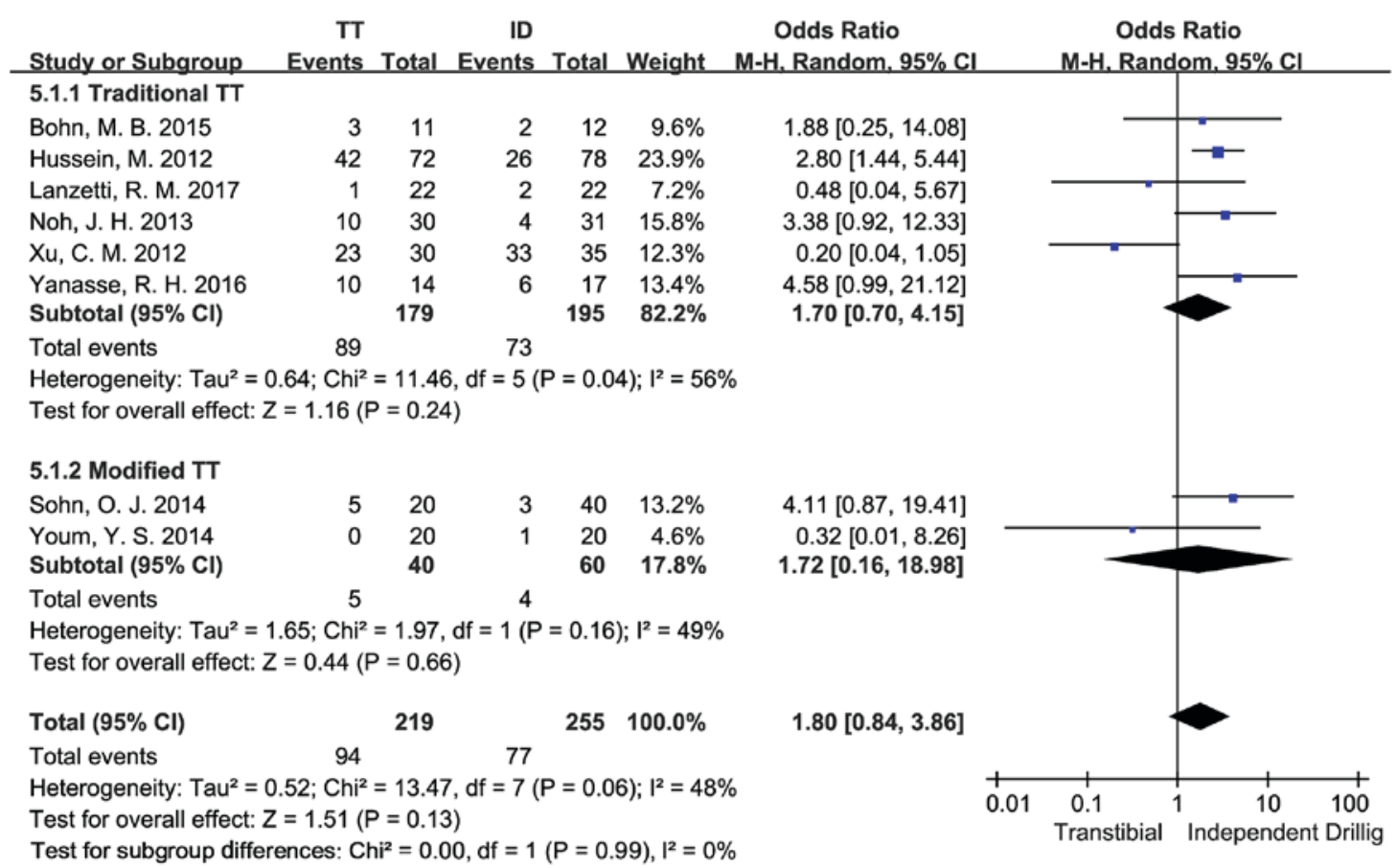

Figure 6. Comparison of pivot-shift test results between the TT and the ID group. TT, transtibial; ID, independent drilling.

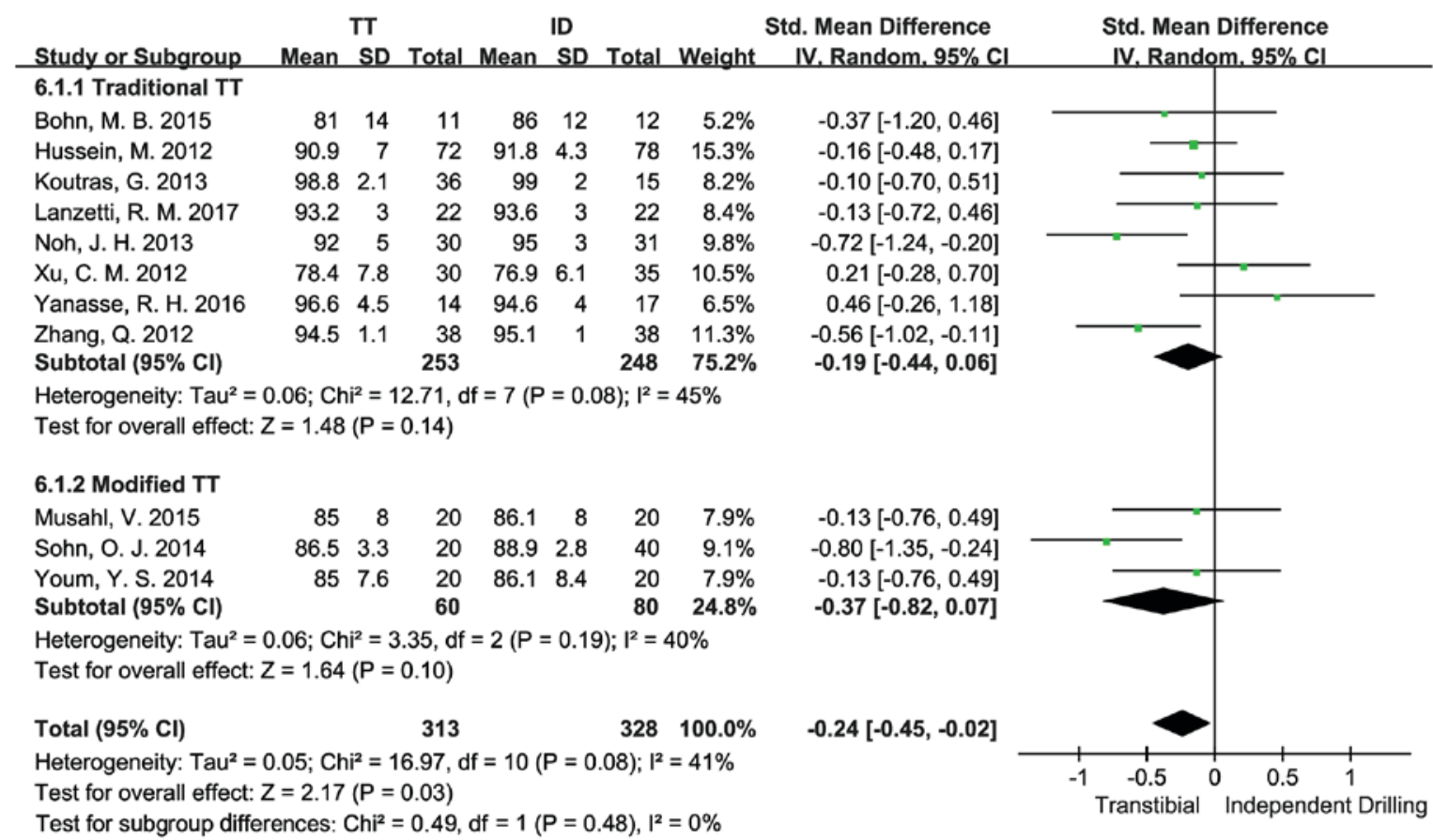

Figure 7. Comparison of Lysholm knee scores between the TT and the ID group. TT, transtibial; ID, independent drilling.

included in the comparison between the sTT and the ID techniques. According to the study results, no statistically significant differences were seen in Tegner activity scale between the sTT and the ID group [SMD $=-0.22,95 \% \mathrm{CI}$ $(-1.16,0.73), \mathrm{P}=0.66]$. The data showed high heterogeneity $\left(\mathrm{P}=0.006, \mathrm{I}^{2}=81 \%\right)$. Besides, there were 140 patients in three studies $(15,17,23)$ included in the comparison between the mTT and the ID techniques. The results indicated no statistically significant differences in Tegner activity scale between the mTT and the ID group [SMD $=-0.11,95 \%$ CI $(-0.48,0.25)$,
$\mathrm{P}=0.55]$, with the data showing low heterogeneity $(\mathrm{P}=0.32$, $\mathrm{I}^{2}=13 \%$ ) (Fig. 8).

Susceptibility analysis. A series of susceptibility analysis was performed to assess the stability of this meta-analysis. To accurately identify the reasons for data instability, the susceptibility analysis was further carried out by selecting the included studies with the sample sizes $>20$ cases and randomized controlled trial (RCT), changing the random effects model into the fixed-effects model and ruling out 


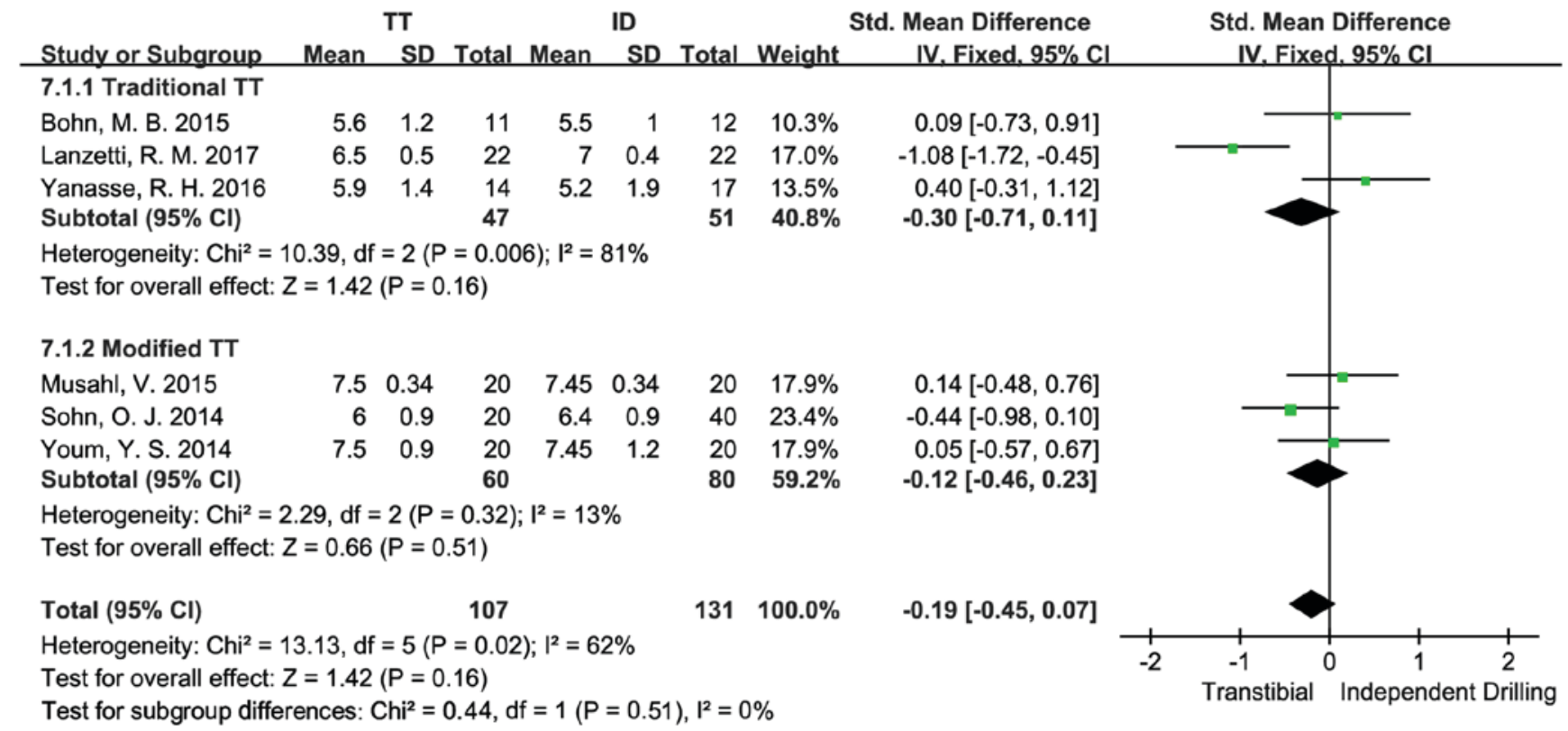

Figure 8. Comparison of Tegner activity scale between the TT and the ID group. TT, transtibial; ID, independent drilling.

singular values. According to the susceptibility analysis on comparison between the sTT and ID techniques, when the studies having smaller sample sizes $(<20$ cases $)$ were excluded, there were statistically significant differences in Lachman test between the sTT and the ID groups [OR=3.19, $95 \%$ CI $(1.01,10.03), \mathrm{P}=0.05]$; if only the studies with RCT were included, the data of the two groups would show significant statistical differences $[\mathrm{OR}=2.39,95 \% \mathrm{CI}(0.90$, 6.35), $\mathrm{P}=0.08]$. In terms of pivot-shift test, if only the studies with RCT were included, the meta-analysis results would suggest significant statistical differences, with the ID group having more negative results $[\mathrm{OR}=2.81,95 \% \mathrm{CI}(1.59,4.96)$, $\mathrm{P}=0.0004]$; when the random effects model applied to the meta-analysis was replaced by the fixed-effects model, statistically significant differences between the data of the two groups could also be found [OR=2.00, 95\% CI (1.25, 3.19), $\mathrm{P}=0.004]$.

In comparison between the sTT and ID techniques, when the article contributed by Matassi et al (21) was removed from the analysis of IKDC subjective score, the data heterogeneity significantly dropped $\left(\mathrm{P}=0.97, \mathrm{I}^{2}=0 \%\right)$ and significant statistical differences between the two groups were observed [SMD $=-0.30,95 \%$ CI $(-0.57,-0.03), \mathrm{P}=0.03]$; as to Lachman test, when Yanasse's et al (22) study was excluded, the analytical results showed statistically significant differences between the two groups [OR=2.39, 95\% $\mathrm{CI}(0.90,6.35), \mathrm{P}=0.08]$, with the ID group having less positive results and without data heterogeneity $\left(\mathrm{P}=0.35, \mathrm{I}^{2}=0 \%\right)$; in terms of pivot-shift test, the analytical results suggested significant statistical differences after the article of $\mathrm{Xu}$ et al (24) was excluded [OR=2.75, 95\% CI $(1.64,4,63), \mathrm{P}=0.0001]$ and meanwhile, the data heterogeneity was significantly reduced $\left(\mathrm{P}=0.63, \mathrm{I}^{2}=0 \%\right)$; when the study of Lanzetti's et al (14) was excluded from the analysis on Tegner activity scale, there remained no statistically significant differences between the two groups [SMD $=0.27,95 \%$ CI $(-0.27$, $0.80), \mathrm{P}=0.33$ ] and yet the data heterogeneity was decreased significantly $\left(\mathrm{P}=0.57, \mathrm{I}^{2}=0 \%\right)$ (Table II).
In comparison between the mTT and ID techniques, considering the limited literature, only the susceptibility analysis that replaced the random effects model with the fixed-effects model was conducted (Table III). In terms of Lysholm knee score, there were statistically significant intergroup differences when the fixed-effects model was applied to the analysis $[\mathrm{SMD}=-0.39,95 \% \mathrm{CI}(-0.03,-0.74), \mathrm{P}=-0.03]$. The susceptibility analysis on other items showed no significant changes in the meta-analysis results (SMDs or ORs) and statistical significance (P-value). Only the subgroups of a study were not given any susceptibility analysis.

\section{Discussion}

The latest meta-analysis based on the 12 articles showed that the ID techniques, compared to the sTT technique, presented a higher IKDC subjective score and a lower degree of laxity. However, no significant differences were found in the results of the IKDC objective score, pivot-shift test and Lachman test; the comparison of the above-mentioned indicators between the mTT and the ID techniques also suggested no significant differences.

Compared to the sTT group, the ID group had a lower degree of laxity. The data showed low heterogeneity. Authors of this report considered it as a result of the non-anatomic site of the bone tunnel. In contrast, Daniel (27) reported that the differences in anterior displacement of the tibia between bilateral knees of $95 \%$ of those having intact knee joints remained $<3 \mathrm{~mm}$ and they held that the difference in anterior laxity between bilateral knees $>3 \mathrm{~mm}$ indicated ACL injuries. In this study, the difference in anterior laxity between bilateral knees was $<3 \mathrm{~mm}$ and yet, it still represented postoperative differences between the two surgical methods. A majority of studies (28-30) indicated that the sTT technique, as non-anatomic reconstruction showing weak anti-rotation ability, was likely to cause impingement of intercondylar fossa and osteoarthritis. The meta-analysis results were in 
Table II. Comparison of susceptibility analysis results between the sTT and the ID techniques.

\begin{tabular}{|c|c|c|c|c|c|c|}
\hline & \multicolumn{3}{|c|}{ IKDC objective score } & \multicolumn{3}{|c|}{ IKDC subjective score } \\
\hline & No. of patients & OR & $\mathrm{P}$-value & No. of patients & SMD & P-value \\
\hline Basic analysis & 305 & $0.48(0.11,2.14)$ & 0.34 & 257 & $-0.48(-0.86,-0.10)$ & 0.01 \\
\hline$n \geq 20$ & 251 & $0.24(0.04,1.53)$ & 0.13 & 234 & $-0.53(-1.02,-0.04)$ & 0.03 \\
\hline $\mathrm{RCT}$ & 234 & $0.24(0.04,1.53)$ & 0.13 & 173 & $-0.28(-0.58,0.02)$ & 0.06 \\
\hline Fixed-effects model & 305 & $0.47(0.12,1.80)$ & 0.27 & 257 & $-0.41(-0.66,-0.16)$ & 0.001 \\
\hline \multirow[t]{3}{*}{ Singular value removal } & I & 1 & l & 217 & $-0.30(-0.57,-0.03)$ & 0.03 \\
\hline & \multicolumn{3}{|c|}{ Knee laxity } & \multicolumn{3}{|c|}{ Lachman test } \\
\hline & No. of patients & SMD & $\mathrm{P}$-value & No. of patients & OR & P-value \\
\hline Basic analysis & 398 & $0.36(0.16,0.56)$ & 0.0004 & 159 & $1.72(0.74,3.97)$ & 0.21 \\
\hline$n \geq 20$ & 375 & $0.37(0.17,0.58)$ & 0.0004 & 105 & $3.19(1.01,10.03)$ & 0.05 \\
\hline $\mathrm{RCT}$ & 249 & $0.35(0.10,0.60)$ & 0.006 & 84 & $2.39(0.90,6.35)$ & 0.08 \\
\hline Fixed-effects model & 398 & $0.36(0.16,0.56)$ & 0.0004 & 159 & $1.75(0.80,3.83)$ & 0.16 \\
\hline \multirow[t]{3}{*}{ Singular value removal } & / & 1 & I & 159 & $1.72(0.74,3.97)$ & 0.21 \\
\hline & \multicolumn{3}{|c|}{ Pivot-shift test } & \multicolumn{3}{|c|}{ Lysholm knee score } \\
\hline & No. of patients & OR & P-value & No. of patients & SMD & $\mathrm{P}$-value \\
\hline Basic analysis & 374 & $1.70(0.70,4.15)$ & 0.24 & 501 & $-0.19(-0.44,0.06)$ & 0.14 \\
\hline$n \geq 20$ & 320 & $1.22(0.34,4.36)$ & 0.76 & 396 & $-0.27(-0.58,0.04)$ & 0.09 \\
\hline $\mathrm{RCT}$ & 234 & $2.81(1.59,4.96)$ & 0.0004 & 310 & $-0.41(-0.69,-0.13)$ & 0.004 \\
\hline Fixed-effects model & 374 & $2.00(1.25,3.19)$ & 0.004 & 501 & $-0.20(-0.38,-0.02)$ & 0.03 \\
\hline Singular value removal & 309 & $2.75(1.64,4.63)$ & 0.0001 & l & / & / \\
\hline
\end{tabular}

Tegner activity scale

\begin{tabular}{lccc} 
& No. of Patients & SMD & P-value \\
\cline { 2 - 4 } Basic analysis & 98 & $-0.22(-1.16,0.73)$ & 0.66 \\
$\mathrm{n} \geq 20$ & $/$ & $/$ & $/$ \\
RCT & $/$ & $/$ & 0.16 \\
Fixed-effects model & 98 & $-0.30(-0.71,0.11)$ & 0.33 \\
Singular value removal & 54 & $0.27(-0.27,0.80)$ & \\
\hline
\end{tabular}

sTT, standard transtibial; ID, independent drilling; IKDC, International Knee Documentation Committee; OR, odds ratio; SMD standardized mean difference; RCT, randomized controlled trial.

Table III. Comparison of susceptibility analysis results between the mTT and the ID techniques.

\begin{tabular}{llc}
\hline & Fixed-effects model & P-value \\
\hline IKDC subjective score & $-0.22(-0.56,0.12)$ & 0.20 \\
Lysholm knee score & $-0.39(-0.73,-0.04)$ & 0.03 \\
Tegner activity scale & $-0.11(-0.48,0.25)$ & 0.55 \\
Pivot-shift test & $2.24(0.61,8.17)$ & 0.22 \\
\hline
\end{tabular}

mTT, modified transtibial; ID, independent drilling; IKDC, International Knee Documentation Committee. line with this theory and explained the reasons why anatomic reconstruction has the advantage over the traditional isometric reconstruction.

Compared to the sTT group, the ID group had a high IKDC subjective score, which showed moderate heterogeneity. The higher IKDC subjective score indicated a higher level of functions or a lower degree of symptoms, with the emphasis placed on the assessment of a patient's range of movement and exercise intensity, instead of the patient's sensations in daily activities, which is the focus of the Lysholm knee scoring scale. The differences in IKDC subjective scores may be affected by the recovery level 
of the physiological structure. Specifically, it is probably because the sTT technique cannot facilitate the recovery of the physiological ligament position that it had a lower IKDC subjective score in the analysis.

As to the comparison of the pivot-shift test, IKDC objective scores and Lachman test, no statistical differences in the ORs were seen between the groups. Moreover, there were few positive results, which indicated that the patients' ACLs had been recovered after operations.

The comparison of IKDC subjective scores, Lysholm knee scores and Tegner activity levels between the mTT and the ID groups showed no significant differences. The mTT technique was put forward by Piasecki et al (31) in 2011. According to their study, anatomic reconstruction could be achieved with the TT technique by changing the knee joint position. Youm et al (23) and Lee et al (32) had put the theory into practice and reported that the TT and IDD techniques were much alike in tunnel characteristics and clinical results. Since the TT technique has been widely accepted in Asia (33-36), it requires further analysis to demonstrate the reliability and benefits of the mTT technique. Therefore, the meta-analysis also provided the comparison between the mTT and ID techniques in order to explore whether the mTT technique could present desirable clinical outcomes. The results suggested that the mTT technique was comparable to the ID techniques in terms of patients' subjective sensations, recovery level of motor abilities and daily activities by comparison of IKDC subjective scores, Lysholm knee scores and Tegner activity levels between the mTT and the ID groups. However, considering the limited data size and short follow-up visit, it still requires massive clinical data to support the conclusion.

In this report, only the single-bundle studies were analyzed because according to the autopsy studies (37) in southern China, the average length of tibial insertions was $15.18 \pm 2.65 \mathrm{~mm}$ only, failing to meet the indication of double-bundle reconstruction. Generally, patients who have the tibial insertions $<16 \mathrm{~mm}$ are recommended to receive single-bundle anatomic reconstruction (38-40).

Similar to other system evaluations, this study also has limitations: i) it includes a small number of RCTs and lacks quality randomized controlled studies; ii) some studies included in the meta-analysis have modest sample sizes and there is statistically significant heterogeneity due to the differences in research design, patient diversity and operation plans; iii) the studies having relatively short follow-up fail to provide long-term clinical evidence; iv) there are few studies on comparison between the mTT and ID techniques written in Chinese, making the meta-analysis less convincing; and v) the meta-analysis is only a statistical test subject to numerous methods and thus it cannot control all relevant factors.

In conclusion, compared to the sTT technique, the mTT technique, similar to the ID techniques, has a lower level of laxity and a higher IKDC subjective score. Since the TT technique is familiar to almost all surgeons, the mTT technique is expected to gain widespread recognition and become the new reconstruction standard in the future. Yet, it still requires extensive research data to support the conclusion.

\section{Acknowledgements}

Not applicable.

\section{Funding}

No funding was received.

\section{Availability of data and materials}

All data generated or analyzed during this study are included in this published article.

\section{Authors' contributions}

$\mathrm{ZY}$ was responsible for the conception and design of the study. QZ and YK were responsible for data collection/entry, data analysis/statistics, data interpretation and literature analysis/search. ZY and QZ were responsible for the preparation of the manuscript. All authors have read and approved the final manuscript.

\section{Ethics approval and consent to participate}

Not applicable.

\section{Patient consent for publication}

Not applicable.

\section{Competing interests}

The authors declare that they have no competing interests.

\section{References}

1. Nebelung W and Wuschech H: Thirty-five years of follow-up of anterior cruciate ligament-deficient knees in high-level athletes. Arthroscopy 21: 696-702, 2005.

2. Brown CH Jr and Carson EW: Revision anterior cruciate ligament surgery. Clin Sports Med 18: 109-171, 1999.

3. Harner CD, Giffin JR, Dunteman RC, Annunziata CC and Friedman MJ: Evaluation and treatment of recurrent instability after anterior cruciate ligament reconstruction. Instr Course Lect 50: 463-474, 2001.

4. Riboh JC, Hasselblad V, Godin JA and Mather RC III: Transtibial versus independent drilling techniques for anterior cruciate ligament reconstruction: A systematic review, metaanalysis, and meta-regression. Am J Sports Med 41: 2693-2702, 2013.

5. Tompkins M, Milewski MD, Brockmeier SF, Gaskin CM, Hart JM and Miller MD: Anatomic femoral tunnel drilling in anterior cruciate ligament reconstruction: Use of an accessory medial portal versus traditional transtibial drilling. Am J Sports Med 40: 1313-1321, 2012.

6. Robin BN and Lubowitz JH: Disadvantages and advantages of transtibial technique for creating the anterior cruciate ligament femoral socket. J Knee Surg 27: 327-330, 2014.

7. Webster KE, Palazzolo SE, McClelland JA and Feller JA: Tibial rotation during pivoting in anterior cruciate ligament reconstructed knees using a single bundle technique. Clin Biomech (Bristol, Avon) 27: 480-484, 2012.

8. Zhang Y, Xu C, Dong S, Shen P, Su W and Zhao J: Systemic review of anatomic single- versus double-bundle anterior cruciate ligament reconstruction: Does femoral tunnel drilling technique matter? Arthroscopy 32: 1887-1904, 2016. 
9. Duquin TR, Wind WM, Fineberg MS, Smolinski RJ and Buyea CM: Current trends in anterior cruciate ligament reconstruction. J Knee Surg 22: 7-12, 2009.

10. Chechik O, Amar E, Khashan M, Lador R, Eyal G and Gold A: An international survey on anterior cruciate ligament reconstruction practices. Int Orthop 37: 201-206, 2013.

11. McRae SM, Chahal J, Leiter JR, Marx RG and Macdonald PB: Survey study of members of the Canadian Orthopaedic Association on the natural history and treatment of anterior cruciate ligament injury. Clin J Sport Med 21: 249-258, 2011.

12. Lee SR, Jang HW, Lee DW, Nam SW, Ha JK and Kim JG: Evaluation of femoral tunnel positioning using 3-dimensional computed tomography and radiographs after single bundle anterior cruciate ligament reconstruction with modified transtibial technique. Clin Orthop Surg 5: 188-194, 2013.

13. Haro MS, Riff A and Bach BR Jr: Tips for successful transtibial anterior cruciate ligament reconstruction. J Knee Surg 27: 331-342, 2014.

14. Lanzetti RM, Lupariello D, De Carli A, Monaco E, Guzzini M, Fabbri M, Vadalà A and Ferretti $\mathrm{A}$ : Can the outside-in halftunnel technique reduce femoral tunnel widening in anterior cruciate ligament reconstruction? A CT study. Eur J Orthop Surg Traumatol 27: 659-664, 2017.

15. Sohn OJ, Lee DC, Park KH and Ahn HS: Comparison of the modified transtibial technique, anteromedial portal technique and outside-in technique in ACL reconstruction. Knee Surg Relat Res 26: 241-248, 2014.

16. Noh JH, Roh YH, Yang BG, Yi SR and Lee SY: Femoral tunnel position on conventional magnetic resonance imaging after anterior cruciate ligament reconstruction in young men: Transtibial technique versus anteromedial portal technique. Arthroscopy 29: 882-890, 2013.

17. Musahl V: A modified transtibial technique was similar to an anteromedial portal technique for anterior cruciate ligament reconstruction. J Bone Joint Surg Am 97: 1373, 2015.

18. Hozo SP, Djulbegovic B and Hozo I: Estimating the mean and variance from the median, range, and the size of a sample. BMC Med Res Methodol 5: 13, 2005.

19. Bohn MB, Sørensen H, Petersen MK, Søballe K and Lind M Rotational laxity after anatomical ACL reconstruction measured by 3-D motion analysis: A prospective randomized clinical trial comparing anatomic and nonanatomic ACL reconstruction techniques. Knee Surg Sports Traumatol Arthrosc 23: 3473-3481, 2015.

20. Hussein M, van Eck CF, Cretnik A, Dinevski D and Fu FH: Prospective randomized clinical evaluation of conventional single-bundle, anatomic single-bundle, and anatomic doublebundle anterior cruciate ligament reconstruction: 281 cases with 3- to 5-year follow-up. Am J Sports Med 40: 512-520, 2012

21. Matassi F, Sirleo L, Carulli C and Innocenti M: Anatomical anterior cruciate ligament reconstruction: Transtibial versus outside-in technique: SIGASCOT Best Paper Award Finalist 2014. Joints 3: 6-14, 2015.

22. Yanasse RH, Lima AA, Antoniassi RS, Ezzedin DA, Laraya MH and Mizobuchi RR: Transtibial technique versus two incisions in anterior cruciate ligament reconstruction: Tunnel positioning, isometricity and functional evaluation. Rev Bras Ortop 51: 274-281, 2016

23. Youm YS, Cho SD, Lee SH and Youn CH: Modified transtibial versus anteromedial portal technique in anatomic single-bundle anterior cruciate ligament reconstruction: Comparison of femoral tunnel position and clinical results. Am J Sports Med 42: 2941-2947, 2014.

24. Xu CM, Yang L, Hu YH and Huangfu XQ: Transplantation of single-bundle four-strand hamstring tendon via anteromedia approach for anterior cruciate ligament reconstruction. Chin J Tissue Eng Res 16: 9975-9979, 2012.

25. Zhang Q, Zhang S, Li R, Liu Y and Cao X: Comparison of two methods of femoral tunnel preparation in single-bundle anterior cruciate ligament reconstruction: A prospective randomized study. Acta Cir Bras 27: 572-576, 2012.
26. Koutras G, Papadopoulos P, Terzidis IP, Gigis I and Pappas E: Short-term functional and clinical outcomes after ACL reconstruction with hamstrings autograft: Transtibial versus anteromedial portal technique. Knee Surg Sports Traumatol Arthrosc 21: 1904-1909, 2013.

27. Daniel DM: Assessing the limits of knee motion. Am J Sports Med 19: 139-147, 1991.

28. Zavras TD, Race A, Bull AM and Amis AA: A comparative study of 'isometric' points for anterior cruciate ligament graft attachment. Knee Surg Sports Traumatol Arthrosc 9: 28-33, 2001.

29. Kilinc BE, Kara A, Oc Y, Celik H, Camur S, Bilgin E, Erten YT, Sahinkaya T and Eren OT: Transtibial vs anatomical single bundle technique for anterior cruciate ligament reconstruction: A Retrospective Cohort Study. Int J Surg 29: 62-69, 2016

30. Franceschi F, Papalia R, Rizzello G, Del Buono A, Maffulli N and Denaro V: Anteromedial portal versus transtibial drilling techniques in anterior cruciate ligament reconstruction: Any clinical relevance? A retrospective comparative study. Arthroscopy 29: 1330-1337, 2013.

31. Piasecki DP, Bach BR Jr, Espinoza Orias AA and Verma NN: Anterior cruciate ligament reconstruction: Can anatomic femoral placement be achieved with a transtibial technique? Am J Sports Med 39: 1306-1315, 2011.

32. Lee JK, Lee S, Seong SC and Lee MC: Anatomic single-bundle ACL reconstruction is possible with use of the modified transtibial technique: A comparison with the anteromedial transportal technique. J Bone Joint Surg Am 96: 664-672, 2014.

33. Misonoo G, Kanamori A, Ida H, Miyakawa S and Ochiai N: Evaluation of tibial rotational stability of single-bundle vs. anatomical double-bundle anterior cruciate ligament reconstruction during a high-demand activity - a quasi-randomized trial. Knee 19: 87-93, 2012.

34. Yagi M, Kuroda R, Nagamune K, Yoshiya S and Kurosaka M: Double-bundle ACL reconstruction can improve rotational stability. Clin Orthop Relat Res 454: 100-107, 2007.

35. Terabe Y, Harada A, Tokuda H, Okuizumi H, Nagaya $M$ and Shimokata H: Vitamin D deficiency in elderly women in nursing homes: Investigation with consideration of decreased activation function from the kidneys. J Am Geriatr Soc 60: 251-255, 2012.

36. Fujita N, Kuroda R, Matsumoto T, Yamaguchi M, Yagi M, Matsumoto A, Kubo S, Matsushita T, Hoshino Y, Nishimoto K, et al: Comparison of the clinical outcome of double-bundle, anteromedial single-bundle, and posterolateral single-bundle anterior cruciate ligament reconstruction using hamstring tendon graft with minimum 2-year follow-up. Arthroscopy 27: 906-913, 2011.

37. Beyaz S, Güler UO, Demir S, Yüksel S, Cınar BM, Özkoc G and Akpınar S: Tunnel widening after single- versus double-bundle anterior cruciate ligament reconstruction: A randomized 8-year follow-up study. Arch Orthop Trauma Surg 137: 1547-1555, 2017.

38. Schreiber VM, van Eck CF and Fu FH: Anatomic Doublebundle ACL Reconstruction. Sports Med Arthrosc Rev 18: 27-32, 2010 .

39. Siebold R: The concept of complete footprint restoration with guidelines for single- and double-bundle ACL reconstruction. Knee Surg Sports Traumatol Arthrosc 19: 699-706, 2011.

40. Chiba D, Tsuda E, Sasaki S, Liu X and Ishibashi Y: Anthropometric and skeletal parameters predict 2-strand semitendinosus tendon size in double-bundle anterior cruciate ligament reconstruction. Orthop J Sports Med 5: 2325967117720148, 2017.

This work is licensed under a Creative Commons Attribution-NonCommercial-NoDerivatives 4.0 International (CC BY-NC-ND 4.0) License. 